\title{
Laser driven structured quantum rings
}

\author{
Giuseppe Castiglia ${ }^{1}$, Pietro Paolo Corso $^{1}$, Umberto De \\ Giovannini $^{2}$, Emilio Fiordilino*1, and Biagio Frusteri ${ }^{1}$ \\ ${ }^{1}$ Dipartimento di Fisica e Chimica, Università degli Studi Palermo, \\ Via Archirafi 36, 90123, Palermo, Italy \\ ${ }^{2}$ DNano-Bio Spectroscopy Group and ETSF Scientific Development \\ Center, Departamento de Quimica, Universidad del País Vasco \\ UPV/EHU, Avenida de Tolosa 72, E-20018, San Sebastián, Spain
}

December 8, 2014

\begin{abstract}
In this work we study harmonic emission from structured quantum rings (SQRs). In SQRs, electrons trapped in two-dimensional structures are further confined by an external potential composed of $N$ scattering centers arranged on a circle. We build a suitable one-dimensional model Hamiltonian describing this class of systems and analytically solve the associated Schödinger equation. We find that the solution can be expressed in terms of Mathieu functions and focus on the specific case of $N=6$. By exactly solving the time-dependent Schödinger equation, we then show how the harmonic response to linearly polarized lasers strongly depends on the ring physical parameters. The results illustrate how the additional degrees of freedom introduced by these parameters, provide important handles to control the emitted spectrum that in some cases extends into the XUV region.
\end{abstract}

\section{Introduction}

High harmonic generation (HHG) is the phenomenon occurring when matter, irradiated by strong lasers, emits light at multiple frequencies of that of the incident pulse. It is a strongly non-linear process that has been extensively studied both experimentally and theoretically. Using HHG as an efficient source of high-frequency coherent radiation is one of the main goals of the research in the field. For this reason, efforts have been made in order to understand, control and manipulate the physical properties (shape, strength, extension, etc.) of the

*emilio.fiordilino@unipa.it 
emitted spectrum. While most of the studies in this direction have been focused on gas-phase atomic targets with optimization techniques based on multicolor pulse shaping and/or phase matching [1], much less has been done towards the controls offered by changing the physical properties of each single emitter.

Atomic systems, which are by far the most studied emitters, offer little space for optimization. Molecular systems with complex electronic structures, on the other hand, provide a lager space to maneuver. As a matter of fact, many works focused on the investigation of HHG from molecules with a wide range of cases spanning from benzene [2,3] and atomic clusters [4] to fullerenes [5, 6, 7, 8, 9, 10] and carbon nanotubes [11]. These works indeed proved molecules to be rich and interesting sources of harmonics. However, applications have been, so far, quite limited. One of the main reasons being that, compared to atomic systems, they present several experimental challenges that need to be overcame in order to become usable sources of radiation.

Artificial atoms and molecules $[12,13,14,15,16,17]$, realized with mesoscopic devices, offer a promising alternative ground of research. Compared to natural molecules these devices present greater flexibility provided by the technological advances in fabrication of low dimensional systems such as twodimensional electron gases, quantum rings and quantum dots. Besides offering the possibility to tailor the physical properties of each single emitter, these technologies allow for the production of multiple devices ordered in spatial arrays thus extending the range of possibilities to engineered phase matching.

To date, research in this direction has been mostly carried out from the theoretical standpoint. Harmonic emission from quantum rings have been investigated in combination with linearly [18] and circularly polarized [19] laser fields. In spite of their simplicity, even in these seemingly simple systems, HHG from circular fields has been predicted to present non-trivial selection rules [19]. Yet, more articulated rules have been demonstrated for benzene model systems [20, 21] and rings composed of silver quantum dots [22]. Compared to real benzene, the artificial molecule has been shown to present characteristic double plateaus in the spectrum [22]. Finally, HHG in the THz range has been studied in mesoscopic rings with a localized impurity [23], and even harmonics production have been investigated in coupled quantum dots [24, 25].

In the present work, we study the harmonic response of rings composed of planar quantum dots to linearly polarized laser fields. Compared to other works in the field we here differentiate by presenting a systematic study of the harmonic spectrum as a function of the ring characteristic parameters: radius, electron effective mass, and angular structure. Flexibility in the change of these variables reflect one of the most important features of these artificial systems that has been so far very little explored. The results obtained from such exploration directly illustrate to which extent it is possible to control features of the harmonic spectrum by engineering the properties of the emitting rings and indicate them as possible sources of XUV radiation. 


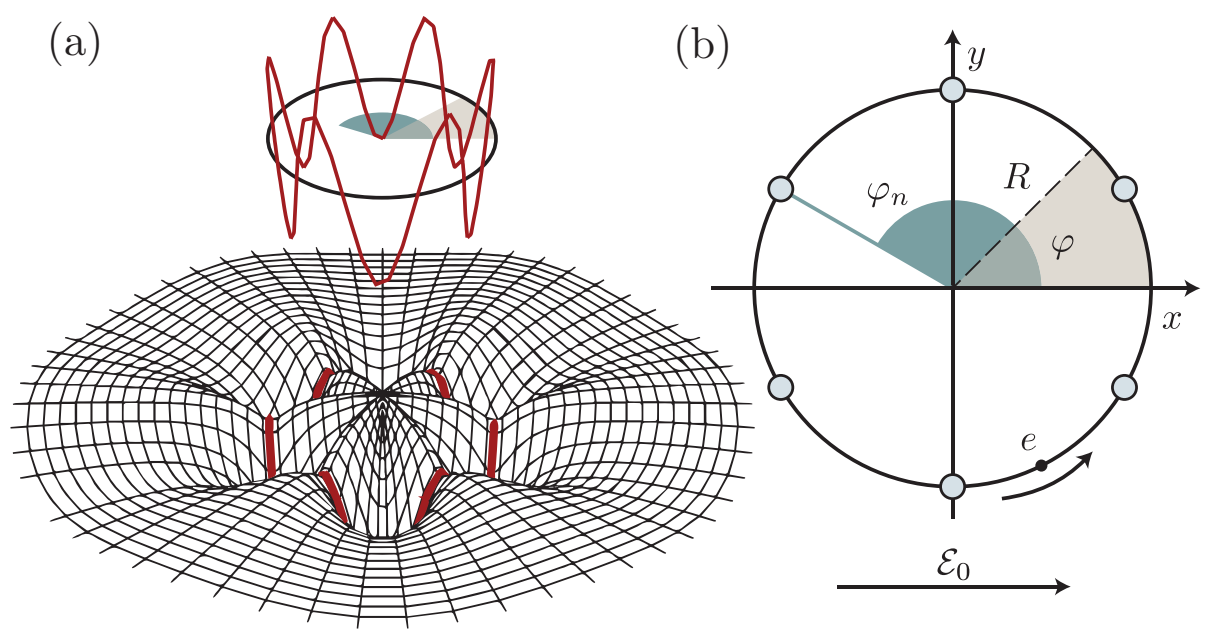

Figure 1: Scheme illustrating the derivation of the model Hamiltonian employed in the preset work. (a) The full potential landscape for a structured two-dimensional quantum ring with $N=6$ centers is approximated with (b) an oscillating potential in one-dimension.

\section{Model}

In this work we focus on SQRs composed of $N=6$ planar quantum dots evenly spaced around a circle. In order to highlight the specific features deriving from the single particle properties of these systems we restrict ourselves to the case of rings containing a single electron only. This situation can be easily achieved under experimental conditions where the charge status of each dot can be precisely tuned, for instance, by electrostatic gating.

In these structures, an electron is effectively constrained in two-dimensions and subject to a multi-center electrostatic potential as depicted in Fig. 1 (a). Instead of addressing the problem containing the full potential we here derive a simplified model still containing the essential physical properties of the original one.

In polar coordinates $(r, \varphi)$, the static Coulomb potential felt by the electron can be written as

$$
V_{s}(r, \varphi)=-\sum_{n=1}^{6} \frac{e Q}{\sqrt{r^{2}+R^{2}+\epsilon-2 R r \cos \left(\varphi-\varphi_{n}\right)}}
$$

where $\epsilon$ is a soft-core smoothing constant accounting for the effective charge densities representing each dot. Such densities are normalized to $Q$ and placed at $\varphi_{n}$ (with angular spacing $\pi / 3$ ).

In a first approximation, an electron subject to the potential of Eq. (1) is constrained in the radial direction and mainly localized around the ring radius. We thus fix $r=R$, and highlight the 6 -fold periodic symmetry expanding in 
Fourier components along the angular coordinate $\varphi$

$$
V_{s}(r=R, \varphi)=-\frac{e Q}{\sqrt{2} R} \sum_{k=0}^{\infty} A_{k} \cos (6 k \varphi) .
$$

The two-dimensional problem is thus reduced to a one-dimensional one as illustrated in Fig. 1. The one-electron effective Hamiltonian therefore becomes

$$
H_{s}=\frac{L_{z}^{2}}{2 \mu R^{2}}-\frac{e Q}{\sqrt{2} R} \sum_{k=1}^{\infty} A_{k} \cos (6 k \varphi)
$$

with $L_{z}=-i \hbar \partial / \partial \varphi$ being the angular momentum operator along $z$, the axis perpendicular to the ring plane, and $\mu$ the effective mass of the charge carrier. Due to the presence of the angular structure, $L_{z}$ is not a conserved quantity and the Schrödinger equation assumes the form of an Hill's differential equation [26].

The periodic potential of Eq. (2) can be further truncated at the first order and the constant term for $k=0$, representing a mere shift of the total energy, can be safely dropped. With this truncated potential, the Hamiltonian finally becomes

$$
H_{0}=\frac{L_{z}^{2}}{2 \mu R^{2}}+\hbar \Omega_{0} \cos (6 \varphi)
$$

thus equivalent to the one of a single electron in 1D with periodic boundary conditions and subject to an oscillating potential that parametrically depends on $\Omega_{0}$.

The stationary Schrödinger equation associated with this Hamiltonian can be recast in the form of a Mathieu's equation

$$
\frac{d^{2} u_{n}}{d x^{2}}+\left[a_{q}^{(n)}-2 q \cos (2 x)\right] u_{n}(x)=0
$$

with a simple change of variable $x=3 \varphi(x \in[0,6 \pi])$ together with the condition $u_{n}(0)=u_{n}(6 \pi)$, and by defining the auxiliary parameters

$$
q=\frac{\mu R^{2} \Omega_{0}}{6^{2} \hbar}, \quad a_{q}^{(n)}=\frac{2 \mu R^{2} E_{n}}{6^{2} \hbar^{2}} .
$$

Here $n$ is an integer $(n \geq 0)$ that serves as principal quantum number and that identifies a Mathieu eigenvalue $a_{q}^{(n)}$ which, in turn, is connected with each eigenvalue $E_{n}$ of the Schrödinger equation.

Solutions of Eq. (5) have to be searched in the class of functions having period $6 \pi$. While Mathieu's equation with $\pi$ and $2 \pi$ periodicities has been extensively studied in the literature $[27,28,29,30,31,32]$, very little or no information can be found for the $6 \pi$ case. For this reason, solving Eq. (5) goes beyond the simple characterization of our specific physical model and assumes a more general scope. In what follows we thus briefly discuss the salient features of the resulting solutions. 

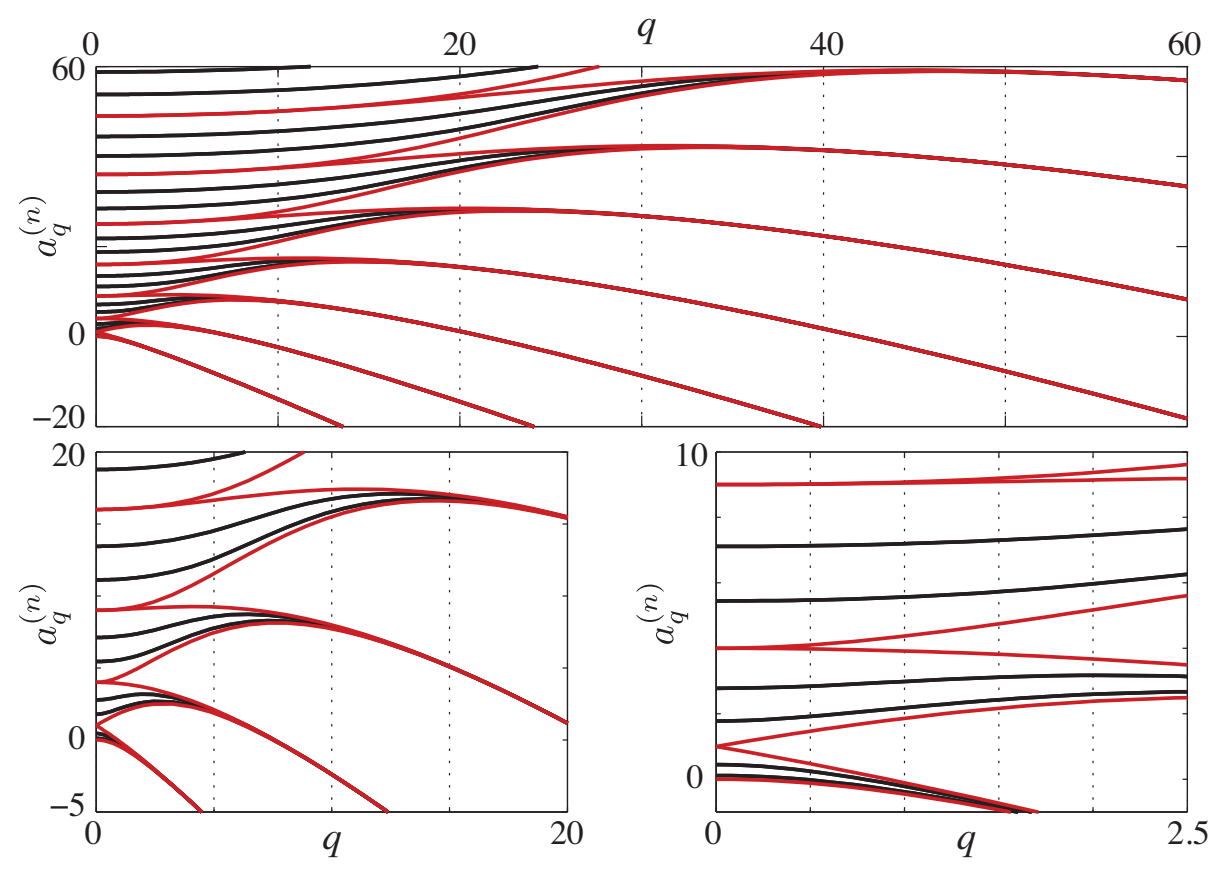

Figure 2: Mathieu equation eigenvalue spectrum $a_{q}^{(n)}$ as a function of $q$ as defined in Eq. (6). Lower panels zoom into different regions of the upper one. Red lines represent non-degenerate eigenvalues while black lines correspond to two-fold degenerate eigenvalues.

For any given eigenvalue $a_{q}^{(n)}$, Eq. (5) may admits two linearly independent eigenstates [31] with the following properties: (i) all solutions have a well defined parity; (ii) if one of the solutions does not have period equal to $\pi$ or to $2 \pi$ both are then degenerate and periodic with opposite parity; (iii) if one of the two solutions has period equal to $\pi$ or to $2 \pi$ the other one cannot be periodic. This last solution can then be discarded as nonphysical since it does not satisfy the $6 \pi$ periodicity condition. On the other hand, solutions with periodicity $6 \pi$ admit simultaneous periods $\pi$ or $2 \pi$.

Owing to (i), for a given $n$, we may have an even and/or an odd solution indicated with ${ }^{e} u_{n}(x)$ and ${ }^{o} u_{n}(x)$ respectively. These eigenfunctions can be arranged into groups pertaining to four sequential values of $n$. Solutions with $n=4 j$ and $n=4 j-1(j=0,1, \ldots)$ are non-degenerate and with opposite parity: ${ }^{e} u_{4 j}(x)$ and ${ }^{o} u_{4 j-1}(x)$. Solutions with $n=4 j+1$ and $n=4 j+2(j=$ $0,1, \ldots)$ are two-fold degenerate with opposite parity: ${ }^{o} u_{4 j+1}(x),{ }^{e} u_{4 j+1}(x)$ and ${ }^{o} u_{4 j+2}(x),{ }^{e} u_{4 j+2}(x)$. The lowest eigenstate has even symmetry ${ }^{e} u_{0}(x)$. These considerations allow to order each group into six eigenstates with the following properties: the first and the last members of the group are non-degenerate with opposite parity (according to the previous discussion with period $\pi$ or $2 \pi$ and respective submultiples), while second with third, and fourth with fifth are degenerate with opposite parity. 
Further degeneracies can be induced by changing $q$ as shown in Fig. 2, where we plot the eigenvalue spectrum $a_{q}^{(n)}$ as a function of this parameter. For small values of $q$ (lower right panel) the spectrum presents an alternating pattern of two-fold degenerate (black) and non-degenerate (red) levels. In the limit $q \rightarrow 0$, Eq. (5) describes a ring with no angular potential. All the non-degenerate levels except the lowest one $(n=0)$ becomes thus degenerate and reproduce the spectrum of a plain ring [18]. In the opposite limit (upper panel in the figure), we observe the formation of degenerate branches composed by levels belonging to the same six element group counting two two-fold degenerate states and two non-degenerate ones.

In order to study the harmonic response of our system we introduce an external laser field linearly polarized along the $x$ axis as in Fig. 1 (b). In dipole approximation the Hamiltonian becomes

$$
H(t)=H_{0}+R \mathcal{E}_{0} f(t) \cos (\varphi) \sin \left(\omega_{L} t\right)
$$

where $\omega_{L}$ and $f(t)$ represent the laser carrier frequency and pulse shape respectively. The time-evolution from the initial ground-state wavefunction can be obtained by numerical integration of the the time dependent Schrödinger equation (TDSE). From the evolving wavefunction it is straightforward to calculate the time-dependent dipole moment $d(t)$ coupling with an external fields. The harmonic response, is directly connected to the dipole acceleration $a(t)=$ $\partial^{2} d(t) / \partial t^{2}$ as its Fourier transform: $I(\omega) \propto|\tilde{a}(\omega)|^{2}$.

\section{Results and comments}

Besides the variables pertaining to the external field, the Hamiltonian governing the dynamics of a SQR given by Eq. (7) depends on several geometric parameters. The ability to tune such parameters at will is a fundamental feature of these systems that have a strong impact on the harmonic response. Below we present a detailed study of this dependence.

To this end we discretize the space and time coordinates and numerically propagate the TDSE with a split-step time evolution operator. To better focus on the degrees of freedom offered by the ring, unless otherwise indicated, we fix the external field to be a 32 cycles ( 2 cycles ramp) trapezoidal shape laser pulse with $\lambda=1064 \mathrm{~nm}\left(\omega_{L}=1.17 \mathrm{eV}\right)$ and intensity $I=4 \times 10^{14} \mathrm{~W} / \mathrm{cm}^{-2}$.

We begin studying the dependency of the harmonic spectrum $I(\omega)$ as a function of the angular potential strength $\Omega_{0}$ in Fig. 3 ; the ring has $\mu=m_{e}$ and radius $R=5 a_{0}$, where $a_{0}$ is the Bohr radius, and $m_{e}$ the electron mass. Compared with a plain ring (i.e. without angular structure) $\hbar \Omega=0 e^{2} / a_{0}$ the presence of a weak potential such as $\hbar \Omega=0.33 e^{2} / a_{0}$ results in an increase of the harmonic yield and an extension of the maximum emitted frequency. This enhancement, is not homogeneous and, if $\Omega_{0}$ is further increased, the resulting yield decreases while the highest harmonics keep increasing. A similar behavior can be observed for a different ring with $R=10 a_{0}$ as shown in Fig. 4. We

chose here an effective mass of $\mu=0.067 m_{e}$ which is compatible with the 


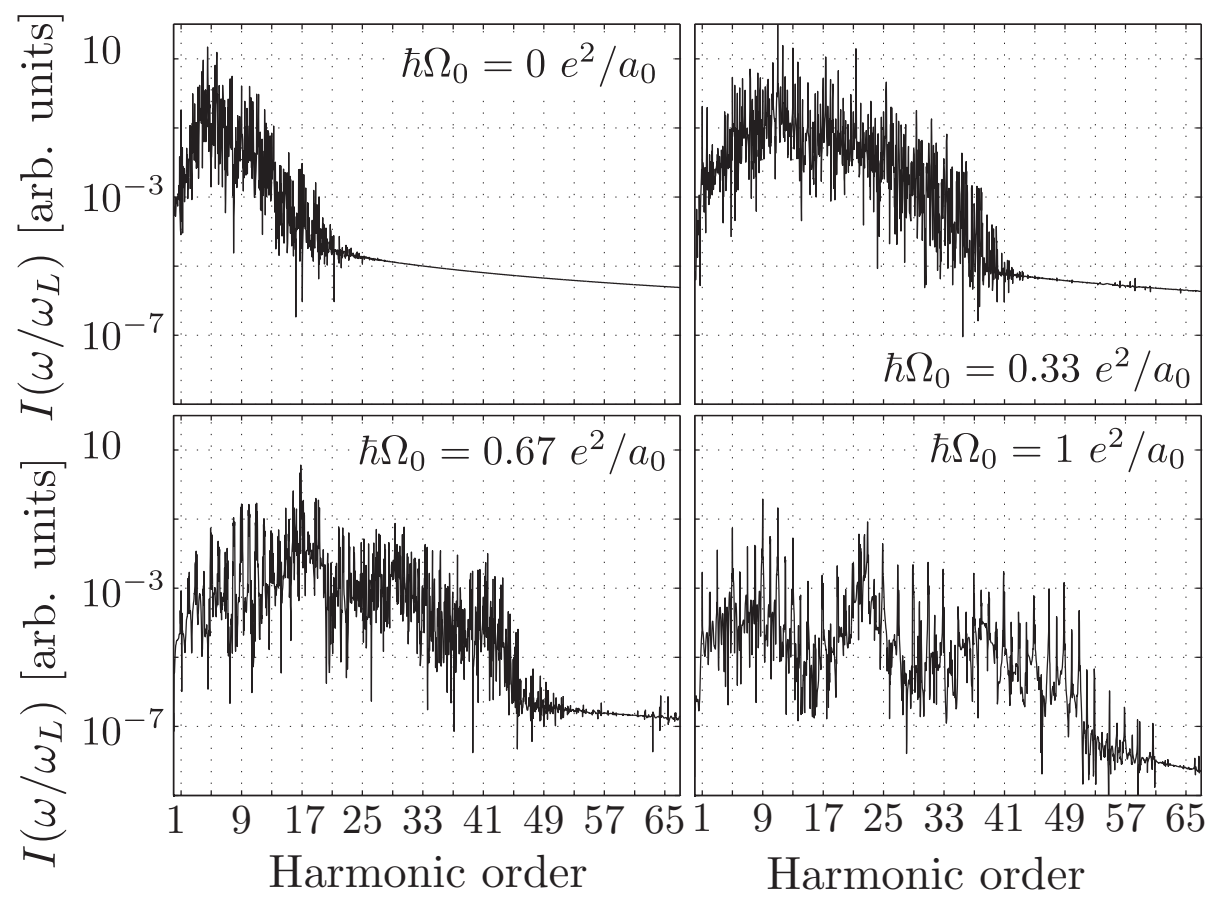

Figure 3: Harmonic spectra $I\left(\omega / \omega_{L}\right)$ (arbitrary units) emitted by a one-electron SQR with $\mu=m_{e}$ and $R=5 a_{0}$ for increasing values of the angular potential strength: (a) $\hbar \Omega=0 e^{2} / a_{0}$, (b) $\hbar \Omega=0.33 e^{2} / a_{0}$, (c) $\hbar \Omega=0,67 e^{2} / a_{0}$, (d) $\hbar \Omega=1 e^{2} / a_{0}$. The ring is irradiated by a 32 cycles, $\lambda=1064 \mathrm{~nm}\left(\omega_{L}=1.17 \mathrm{eV}\right)$, laser pulse of intensity $I=4 \times 10^{14} \mathrm{~W} / \mathrm{cm}^{-2}$ and trapezoidal shape (2 ramp cycles). 


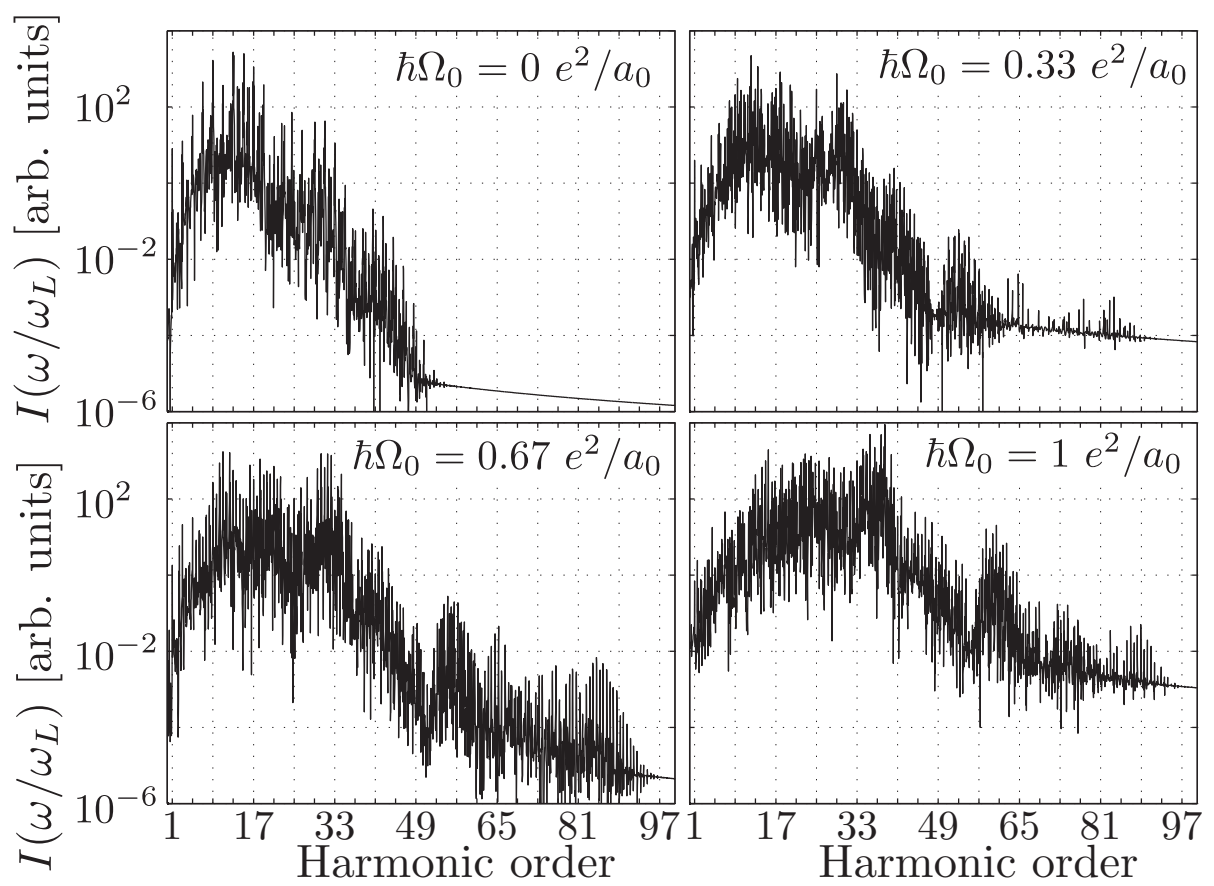

Figure 4: Same as in Fig. 3 but for a ring with $R=10 a_{0}$ and GaAs electron's effective mass $\mu=0.067 m_{e}$. 


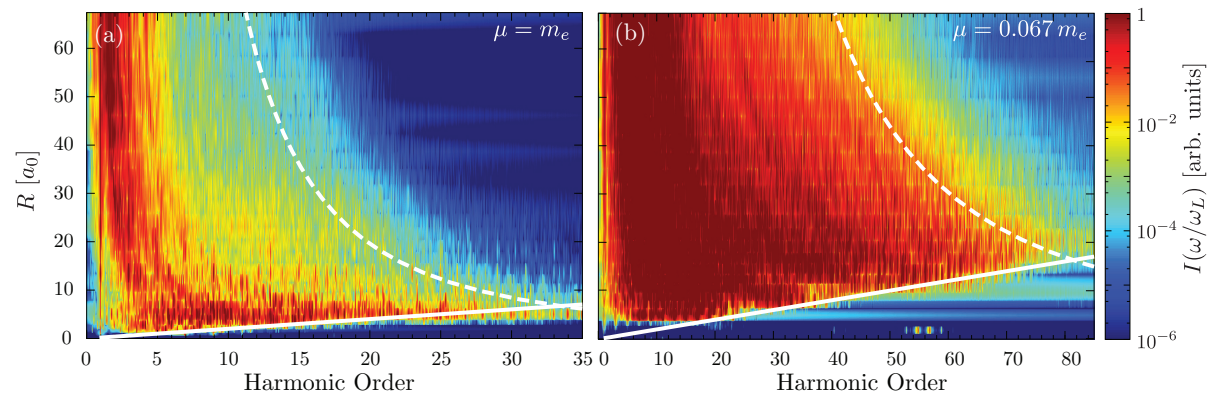

Figure 5: Harmonic spectra (log scale) generated by a SQR as a function of the radius $R$. The effective mass is varied from (a) $\mu=1 m_{e}$ to (b) $\mu=0.067 m_{e}$, while the angular structure paremeter is kept fixed at $\hbar \Omega_{0}=0.33 e^{2} / a_{0}$. White lines indicate the cutoff formulae of Eq. (11) with $A=3.6$ (dashed) and Eq. (10) (solid). The laser parameters are the same as in Fig. 3.

charge carriers in GaAs. Even tough in a less marked way, also in this case the dependency of the emission is not homogeneously increasing with $\Omega_{0}$. Insight on this behavior can be obtained resorting to a classical model of the dynamics.

By solving the canonical equations associated with the classical counterpart of the Hamiltonian in Eq. (7) it is easy to derive the equations of motion governing the electronic coordinate $\varphi(t)$ as a function of time

$$
\mu R^{2} \frac{d^{2} \varphi(t)}{d t^{2}}=\Omega_{0} \cos [6 \varphi(t)]+\mathcal{E}_{0} R \sin [\varphi(t)] \sin \left[\omega_{L} t\right] .
$$

This equation is nonlinear at all orders in $\varphi(t)$ and belongs to a class of equations known to give rise to classical chaos [33]. This means that small variations on the initial conditions can lead to dramatic differences in the final trajectories. The observed non homogeneous behavior of the harmonic yield upon changes on $\Omega_{0}$ may be reminiscent of this chaotic influence. Such influence can survive in the quantum realm with small variations of the physical parameters being reflected in very different dynamical regimes.

Interesting features in the harmonic emission can be observed in the dependency on radius and effective mass. In Fig. 5 we show the power spectra as a function of $R$ and $\mu=m_{e}$ in panel (a) and $\mu=0.067 m_{e}$ in panel (b), while the angular potential is kept fixed at $\hbar \Omega_{0}=0.33 e^{2} / a_{0}$.

Changing the size of the ring has considerable effects on the harmonic yield. For rings having $R \lesssim 1 a_{0}$ the response is practically negligible. The emission then linearly increases with $R$ reaching a maximum extension at $R=6.7 a_{0}$ for $\mu=m_{e}\left(R=16.4 a_{0}\right.$ for $\left.\mu=0.067 m_{e}\right)$ and than decreases for larger values. This can be understood by observing that, in the limit of $R \rightarrow \infty$, electrons no longer feel neither the curvature nor the angular potential and can be regarded as free particles. In this limit, the harmonic response is restricted to the fundamental frequency $\omega_{L}$ only, and the yield thus has to decrease.

Additional understanding can be, once again, obtained resorting to a classical model. Similarly to Eq. (8), from Hamilton's equations it is possible to 
derive an equation governing the time evolution of the kinetic energy

$$
\begin{aligned}
K(t)=\quad & K(0)-\Omega_{0} \cos [6 \varphi(t)]-R \mathcal{E}_{0} \sin \left[\omega_{L} t\right] \cos [\varphi(t)] \\
& +R \mathcal{E}_{0} \omega_{L} \int_{0}^{t} \cos [\varphi(\tau)] \cos \left[\omega_{L} \tau\right] d \tau
\end{aligned}
$$

In analogy to the third step of the famous three-step model $[34,35]$, the classical upper-bound for the maximum harmonic emitted can be estimated assuming that the electron releases its entire energy as a single photon. The maximum value of the average kinetic energy $\langle K(t)\rangle$ thus constitute an upper bound for the highest harmonic emitted. It can be obtained estimating the maximum value of the integral in Eq. (9) over a laser period $T_{L}$ for $\Omega_{0}=0$. Under the assumption that for some laser cycle $\varphi(t)$ is almost constant, it is easy to show that this maximum value is $2 / \omega_{L}$. The classical upper bound for the highest harmonic is

$$
\hbar \omega_{C}=\langle K(t)\rangle_{T_{L}}=2 \mathcal{E}_{0} R,
$$

and only depends on the ring radius $R$ and the peak value of the electric field $\mathcal{E}_{0}$. The solid white lines in Fig. 5 depicting Eq. (10) indicate that the classical limit is a good approximation for small values of $R$.

Changing the values of the effective mass has remarkable effects on the spectra. This can be observed comparing Fig. 5 (a) and (b). From the comparison it is apparent how reducing the mass results in larger harmonic plateau. This can be understood observing that, a reduction of the effective mass is associated to smaller inertial values and larger peak accelerations which, in turn, are directly connected with field emission. Such dependence is even clearer in Fig. 6 where we plot the dipole power spectra as a function of $\mu$ for a ring with $R=10 a_{0}$ and $\hbar \Omega_{0}=0.33 e^{2} / a_{0}$.

The dependence of the highest harmonic on $\mu$ can be estimated from Fig. 6 to be proportional to $1 / \sqrt{\mu}$. In the same way, the decay of the cutoff frequency for large $R$ in Fig. 5 can be estimated proportional to $1 / \sqrt{R}$. Combining these observations with the asymptotic condition imposing a response on the fundamental harmonic for $R \rightarrow \infty$, we can deduce a phenomenological formula

$$
\omega_{P}=\frac{A}{\sqrt{\mu R}}+\omega_{L},
$$

where $A$ is a free parameter. The white dashed lines in Fig. 6 and Fig. 5 represent Eq. (11) for $A=3.6$. The formula thus well describes the main features observed in the harmonic response. Furthermore, it also well suited to reproduce the dependence on the laser wavelength in Fig. 7 for different effective mass values.

For rings of radius $\bar{R}$ such that the classical and phenomenological cutoff lines of Eq. (10) and Eq. (11) cross each other we have maximum harmonic emission. In those rings, the highest harmonic emitted can be estimated to be

$$
\bar{\omega} \approx\left(\frac{2 \mathcal{E}_{0} A^{2}}{\mu}\right)^{\frac{1}{3}} \quad, \text { for } \quad \bar{R} \approx\left(\frac{A^{2}}{4 \mathcal{E}_{0}^{2} \mu}\right)^{\frac{1}{3}},
$$




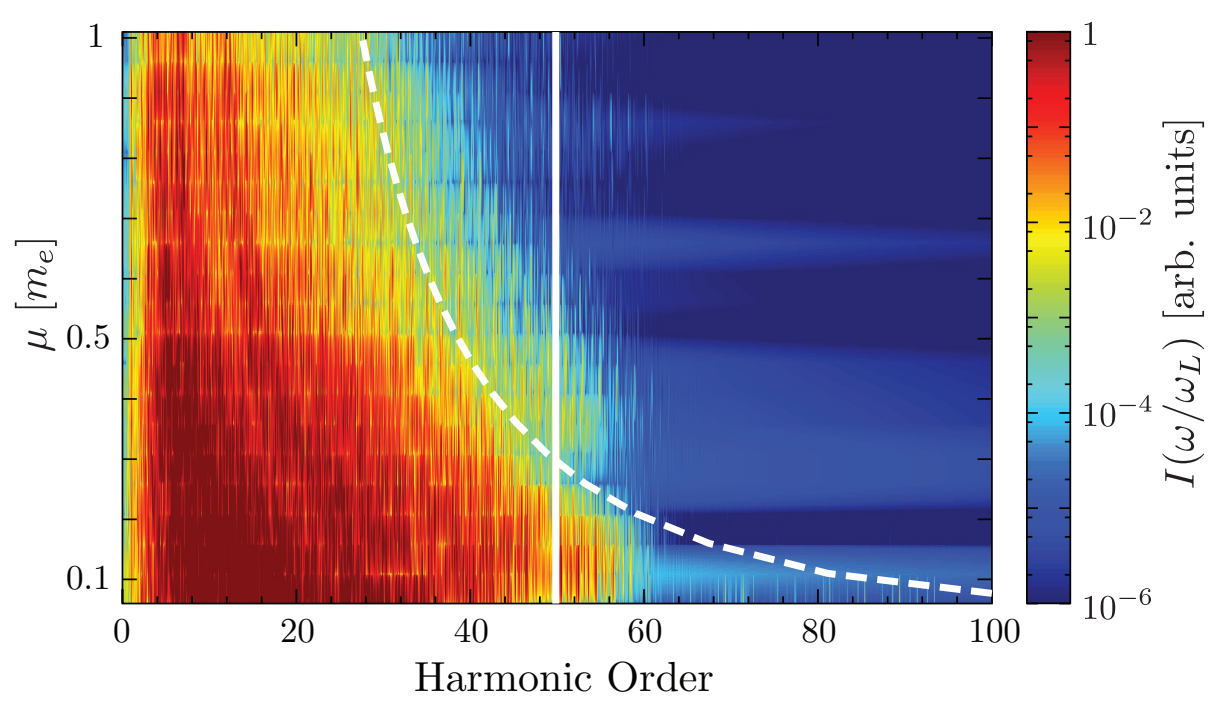

Figure 6: Harmonic power spectrum as a function of the electron effective mass $\mu$ (in units of $m_{e}$ ). The ring has $R=10 a_{0}$ and $\hbar \Omega_{0}=0.33 e^{2} / a_{0}$, while the laser parameters are the same as in Fig. 5. White lines indicate the cutoffs of Eq. (11) with $A=3.6$ (dashed) and Eq. (10) (solid).

and depends on the laser strength and effective mass only. In GaAs the highest emitted harmonic is $\hbar \bar{\omega}=95 \mathrm{eV}$. and can be raised in $\operatorname{InSb}\left(\mu=0.013 m_{e}\right)$ up to $\hbar \bar{\omega}=163 \mathrm{eV}$. The optimal ring size in these cases would be of $\bar{R}=0.9 \mathrm{~nm}$ for GaAs, and $\bar{R}=1.5 \mathrm{~nm}$ for InSb. This fact make SQRs interesting devices for XUV generation.

\section{Conclusions}

In the present work we investigated the harmonic response to intense laser pulses of structured quantum rings (SQRs) composed of six planar quantum dots. We derived a one-dimensional model Hamiltonian describing these systems and found that the associated stationary Schrödinger equation can be identified with as a $6 \pi$ periodicity Mathieu equation.

Compared to atomic and molecular systems SQRs offer additional parameters to control the electron dynamics driven by external fields. To illustrate this feature we investigated the impact of these additional degrees of freedom on HHG.

We showed that the highest harmonic frequency generated presents a maximum for a given radius that moves to higher values as the electron effective mass is reduced. We derived a classical cutoff formula describing the small radius regime and a phenomenological law for larger radii. Finally, by combining the two cutoff laws, we obtained a closed formula characterizing the the highest harmonic and showed that it falls well within the XUV range if the ring is realized in GaAs or InSb. 


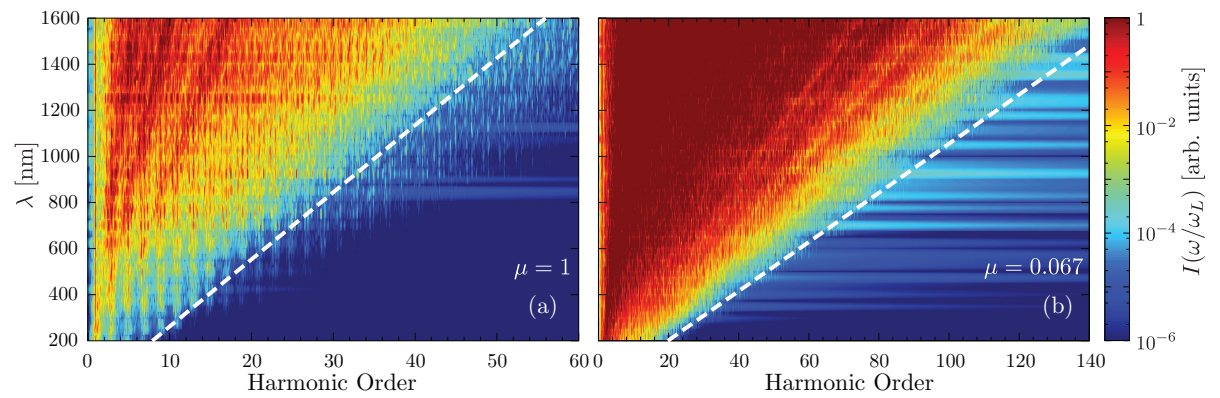

Figure 7: SQR harmonic spectra (log scale) as a function of the laser wavelength. The pulse has peak intensity $I=4 \times 10^{14} \mathrm{~W} / \mathrm{cm}^{-2}$ and a 32 cycles trapezoidal envelope shape with 2 ramp cycles. Effective mass and radius are varied from (a) $\mu=1 m_{e}, R=10 a_{0}$ to (b) $\mu=0.067 m_{e}, R=20 a_{0}$ while $\hbar \Omega_{0}=0.33 e^{2} / a_{0}$. Dashed white lines indicate the cutoff formula of Eq. (11) with $A=3.6$.

The obtained results point out SQRs as interesting harmonic generation devices that can be controlled by engineering their physical properties. In combination with the ability to design their collective properties by creating arrays of emitters would make SQRs attractive sources of XUV radiation. The work presented here constitute a small, but fundamental, step towards the realization of such sources that we hope will stimulate further theoretical and experimental investigation.

\section{Acknowledgments}

We acknowledge financial support from [...]. U.D.G. acknowledges support form the European Commission project CRONOS (Grant number 280879-2 CRONOS CP-FP7). 


\section{References}

[1] C. Winterfeldt, C. Spielmann, and G. Gerber, Rev. Mod. Phys. 80, 117 (2008).

[2] F. Ceccherini and D. Bauer, Phys. Rev. A 64, 033423 (2001).

[3] D. Dundas, J. Chem. Phys. 136, 194303 (2012).

[4] V. Véniard, R. Taïeb, and A. Maquet, Phys. Rev. A 60, 3952 (1999).

[5] M. F. Ciappina, A. Becker, and A. Jaroń-Becker, Phys. Rev. A 78, 063405 (2008).

[6] R. A. Ganeev, L. B. Elouga Bom, M. C. H. Wong, J.-P. Brichta, V. R. Bhardwaj, P. V. Redkin, and T. Ozaki, Phys. Rev. A 80, 043808 (2009).

[7] R. A. Ganeev, L. B. Elouga Bom, J. Abdul-Hadi, M. C. H. Wong, J. P. Brichta, V. R. Bhardwaj, and T. Ozaki, Phys. Rev. Lett. 102, 013903 (2009).

[8] R. A. Ganeev, T. Witting, C. Hutchison, F. Frank, P. V. Redkin, W. A. Okell, D. Y. Lei, T. Roschuk, S. A. Maier, J. P. Marangos, and J. W. G. Tisch, Phys. Rev. A 85, 015807 (2012).

[9] D. Cricchio, E. Fiordilino, and F. Persico, Phys. Rev. A 86, 013201 (2012).

[10] R. A. Ganeev, Optics and Photonics Journal 3, 259 (2013).

[11] R. A. Ganeev, P. A. Naik, H. Singhal, J. A. Chakera, M. Kumar, M. P. Joshi, A. K. Srivastava, and P. D. Gupta, Phys. Rev. A 83, 013820 (2011).

[12] E. Paspalakis, Phys. Rev. B 67, 233306 (2003).

[13] K. K. Gomes, W. Mar, W. Ko, F. Guinea, and H. C. Manoharan Nature 483, 306 (2012).

[14] L. Tarruell, D. Greif, T. Uehlinger, G. Jotzu, and T. Esslinger, Nature 483, 302 (2012).

[15] E. Räsänen, C. A. Rozzi, S. Pittalis, and G. Vignale, Phys. Rev. Lett 108, 246803 (2012).

[16] F. Cavaliere, U. De Giovannini, R. Cenni, M. Sassetti, and B. Kramer, Physica E 40, 1427 (2008).

[17] U. De Giovannini, F. Cavaliere, R. Cenni, M. Sassetti, and B. Kramer, New J. Phys. 9, 93 (2007).

[18] G. Castiglia, P. P. Corso, D. Cricchio, R. Daniele, E. Fiordilino, F. Morales, and F. Persico Phys. Rev. A 88, 033837 (2013). 
[19] O. E. Alon, V. Averbukh, and N. Moiseyev, Phys. Rev. Lett. 80, 3743 (1998).

[20] P. Ždánská, V. Averbukh, and N. Moiseyev, J. Chem. Phys. 118, 8726, (2003).

[21] D. Bauer and F. Ceccherini, Laser and Particle Beams 19, 85 (2001).

[22] I. Bâldea, A. K. Gupta, L. S. Cederbaum, and N. Moiseyev, Phys. Rev. B 69, 245311 (2004).

[23] N. Hinsche, A. Moskalenko, and J. Berakdar, Phys. Rev. A 79, 023822 (2009)

[24] Shi-Fang Guo, Su-Qing Duan, Ning Yang, Wei-Dong Chu, and Wei Zhang, Phys. Rev. A 84, 015803 (2011).

[25] S-F. Guo, S-Q. Duan, Y. Xie, W. Chu, and W. Zhang, New J. Phys 13, 053005 (2011).

[26] E. L. Ince, MNRAS 75, 436 (1915).

[27] E. T. Whittaker, Proc. Edinburgh Math. Soc. 32, 752 (1913).

[28] E. L. Ince, Ordinary Differential Equations, Dover, New York, 1967.

[29] G. B. Arfken and H. J. Weber, Mathematical methods for physicists, Elsevier Academic Press, sixt edition (2005).

[30] P. M. Morse and H. Feshbach, Methods of Theoretical Physics, McGrawHill, New York, 1953.

[31] M. Abramowitz and I. A. Stegun (ed.s), Handbook of Mathematical Functions with Formulas, Graphs, and Mathematical Tables, Dover (1974).

[32] I. S. Gradshteyn and I. M. Ryzhik, Table of Integrals, Series and Products, New York: Academic Press, 7th edition (2007).

[33] J. M. T. Thompson and H. B. Stewart, Nonlinear Dynamics and Chaos, John Wiley \& Sons, LTD (2002).

[34] P. B. Corkum, Phys. Rev. Lett. 71, 1994 (1993).

[35] M. Lewenstein, Ph. Balcou, M. Yu. Ivanov, A. L'Huillier, and P. B. Corkum, Phys. Rev. A 49, 2117 (1994). 